Review Article

\title{
Open versus Closed Kinetic Chain Exercises following an Anterior Cruciate Ligament Reconstruction: A Systematic Review and Meta-Analysis
}

\author{
Daniel Jewiss, ${ }^{1}$ Cecilia Ostman, ${ }^{1}$ and Neil Smart ${ }^{2}$ \\ ${ }^{1}$ School of Rural Medicine, University of New England, Armidale, NSW 2351, Australia \\ ${ }^{2}$ School of Science and Technology, University of New England, Armidale, NSW 2351, Australia \\ Correspondence should be addressed to Neil Smart; nsmart2@une.edu.au
}

Received 8 February 2017; Revised 19 May 2017; Accepted 28 May 2017; Published 17 August 2017

Academic Editor: Ian L. Swaine

Copyright (c) 2017 Daniel Jewiss et al. This is an open access article distributed under the Creative Commons Attribution License, which permits unrestricted use, distribution, and reproduction in any medium, provided the original work is properly cited.

\begin{abstract}
Background. There is no consensus on whether closed kinetic chain (CKC) or open kinetic chain (OKC) exercises should be the intervention of choice following an anterior cruciate ligament (ACL) injury or reconstruction. Methods. A systematic search identified randomized controlled trials of OKC versus CKC exercise training in people who had undergone ACL reconstructive surgery. All published studies in this systematic review were comparisons between OKC and CKC groups. Results. Seven studies were included. Lysholm knee scoring scale was not significantly different between OKC and CKC exercise patients: MD: $-1.03 \%$; CI: $-13.02,10.95 ; p$ value $=0.87\left(\mathrm{Chi}^{2}=0.18, \mathrm{df}=1\right.$, and $p$ value $\left.=0.67\right)$. Hughston clinic questionnaire scores were not significantly different between OKC and CKC exercise patients: $\mathrm{MD}:-1.29 \%(-12.02,9.43) ; p$ value $=0.81\left(\mathrm{Chi}^{2}=0.01, \mathrm{df}=1\right.$, and $p$ value $=$ 0.93). Conclusions. While OKC and CKC may be beneficial during ACL surgical rehabilitation, there is insufficient evidence to suggest that either one is superior to the other.
\end{abstract}

\section{Introduction}

Open kinetic chain (OKC) exercises are lower limb activities performed where the distal segment of the limb is free to move. The opposite of OKC is closed kinetic chain exercises $(\mathrm{CKC})$. There is no consensus among the existing published evidence as to whether closed kinetic chain (CKC) or open kinetic chain (OKC) exercises should be the intervention of choice following an anterior cruciate ligament (ACL) injury or reconstruction. Several outcome measures are recommended to assess ACL injury and rehabilitation outcomes [1]. The commonly held belief has been that OKC exercises cause increased strain on the ACL as well as increased joint laxity and anterior tibial translation [2].

The doubts about the safety of OKC exercises are arguably unsupported by substantial published evidence and are possibly an intuitive opinion. Intuitively, a distally fixed foot in the case of CKC is safer than a nondistally fixed foot in OKC. This stance follows the work of Yack et al., 1993, who showed that there was greater joint laxity using the Anterior Tibial Displacement test during OKC exercises [3]. The assumption was thus made that OKC was more dangerous than CKC given that increased laxity is associated with graft failure and loosening. Beynnon and Fleming (1998) cast doubt as to whether measurable differences in strain between the two forms of exercise exist [4]. Many physiotherapists strongly believe that OKC exercises exert greater strain on the ACL and the patellofemoral joint than CKC exercises [4]. Moreover, the opinion of many physiotherapists is that adverse symptoms such as pain and joint laxity are more likely with OKC exercises than with CKC exercises.

We conducted a systematic analysis of all clinical randomized controlled trials comparing OKC exercises and CKC exercises in patients following ACL reconstruction. We aimed to determine whether there are any differences in clinical outcomes between $\mathrm{OKC}$ and CKC exercise protocols.

\section{Methods}

2.1. Search Strategy. Studies were identified through a MEDLINE search strategy (1966 to October 4, 2016), Cochrane Controlled Trials Registry (1966 to October 4, 2016), 
CINAHL, SPORTdiscus, and Science Citation Index. The search strategy included a mix of MeSH and free text terms for the key concepts related to anterior cruciate ligament reconstruction, exercise training, open chain exercises, and closed chain exercises (see PubMed search strategy in Supplementary Files available online at https://doi.org/10.1155/2017/ 4721548). Studies were included if patients had undergone ACL reconstruction. Searches were limited to prospective randomized or controlled trials in humans. No restrictions were placed on the language of publication. Reference list of papers and latest editions of relevant journals which were not available online were scrutinized for new references. Full articles were read and assessed by two reviewers (C. O. and D. J.) for relevance and study eligibility. Disagreements on methodology were resolved by discussion, and a third reviewer (N. S.) adjudicated over any disputes. Study authors were contacted and requested to provide further data if required.

2.2. Study Selection. Included studies were randomized controlled trials of OKC versus CKC exercise training in people who had undergone ACL reconstructive surgery. All published studies in this systematic review were comparisons between $\mathrm{OKC}$ and $\mathrm{CKC}$ groups.

In addition to the studies identified through database searching, reference lists of identified studies were scrutinized. Only the principal study with the greatest number of subjects was included where multiple publications existed from the same dataset. After initial screening we removed overlapping, duplicates, duplicate data, and irrelevant articles such as editorials and discussion papers that did not match the inclusion criteria. We excluded studies where the subjects had not yet undergone ACL reconstructive surgery, nonrelevant studies, and those reporting only acute exercise testing responses. We excluded studies from specific analyses if incomplete data was reported and the authors did not respond to our requests to provide missing data.

2.3. Outcome Measures. We extracted all possible data; however there were only two outcomes which were reported by more than one paper, the Lysholm knee scoring scale [1] and the Hughston clinic questionnaire [5]. We also recorded exercise training frequency, intensity, duration per session, length of exercise program, participant exercise adherence, and completion rates.

2.4. Data Synthesis. From extracted data we calculated the mean difference for pre/postintervention change in outcome measures and medical events.

2.5. Assessment of Study Quality. We assessed study quality with regard to eligibility criteria specific, random allocation of participants, concealed allocation, similarity of groups at baseline, assessors blinded, outcome measures assessed in $85 \%$ of participants, and intention of treatment analysis. The study quality was assessed according to the validated TESTEX scale which has a maximum score of 15 [6].
2.6. Data Synthesis. Meta-analyses were completed for continuous data by using the change in the mean and standard deviation of outcome measures. It is an accepted practice to only use postintervention data for meta-analysis but this method assumes that random allocation of participants always creates intervention groups matched at baseline for age, disease, severity, and so on. Change in postintervention mean was calculated by subtracting baseline from postintervention values. Data required was either (i) 95\% confidence interval data for pre/postintervention change for each group or, when this was unavailable, (ii) actual $p$ values for pre/postintervention change for each group or, if only the level of statistical significance was available, (iii) default $p$ values; for example, $p<0.05$ becomes $p=0.049, p<0.01$ becomes $p=0.0099$, and $p=$ not significant becomes $p=$ 0.05 .

2.7. Heterogeneity. Clinical heterogeneity was expected given that the data were obtained from patients represented in the included trials. As such, a random effects model was employed. Heterogeneity was quantified using the $I^{2}$ test [7], as it does not inherently depend upon the number of studies considered. $I^{2}$ values range from $0 \%$ (homogeneity) to $100 \%$ (greatest heterogeneity); a CI that does not include $0 \%$ indicates that the hypothesis of homogeneity is rejected, and an inference of heterogeneity is merited [7].

\section{Results}

Our initial search identified 151 studies. After excluding studies based on title and abstract, as well as removing duplicates, 23 studies remained. The full-text articles were assessed for study inclusion, and 16 were excluded (see Table 2); 4 studies were not randomized controlled trials, 6 studies were not postsurgical, and 6 studies did not compare $\mathrm{OKC}$ to $\mathrm{CKC}$ exercise rehabilitation. Seven studies remained, four were included for meta-analysis and three for systematic review only. The search details are provided in the CONSORT statement (Figure 1).

Our analysis of the 4 studies totalled 229 participants: 112 from OKC exercise groups and 117 from CKC exercise groups. Table 1 summarizes the details of the included studies.

\subsection{Meta-Analyses}

3.1.1. Lysholm Knee Scoring Scale. Two studies provided data on the Lysholm knee scoring scale. Results show that there was no significant change in OKC exercise patients versus CKC exercise patients: MD: $-1.03 \%$ and CI: $-13.02,10.95 ; p$ value $=0.87\left(\mathrm{Chi}^{2}=0.18, \mathrm{df}=1\right.$, and $p$ value $=0.67$; between studies variability: $I^{2}=0 \%$ ); see Figure 2 .

3.1.2. Hughston Clinic Questionnaire. Two studies provided data on the Hughston clinic questionnaire. Results show that there was no significant change in OKC exercise patients versus CKC exercise patients: $\mathrm{MD}:-1.29 \%(-12.02,9.43) ; p$ value $=0.81\left(\mathrm{Chi}^{2}=0.01, \mathrm{df}=1\right.$, and $p$ value $=0.93$; between studies variability: $I^{2}=0 \%$ ); see Figure 3 . 


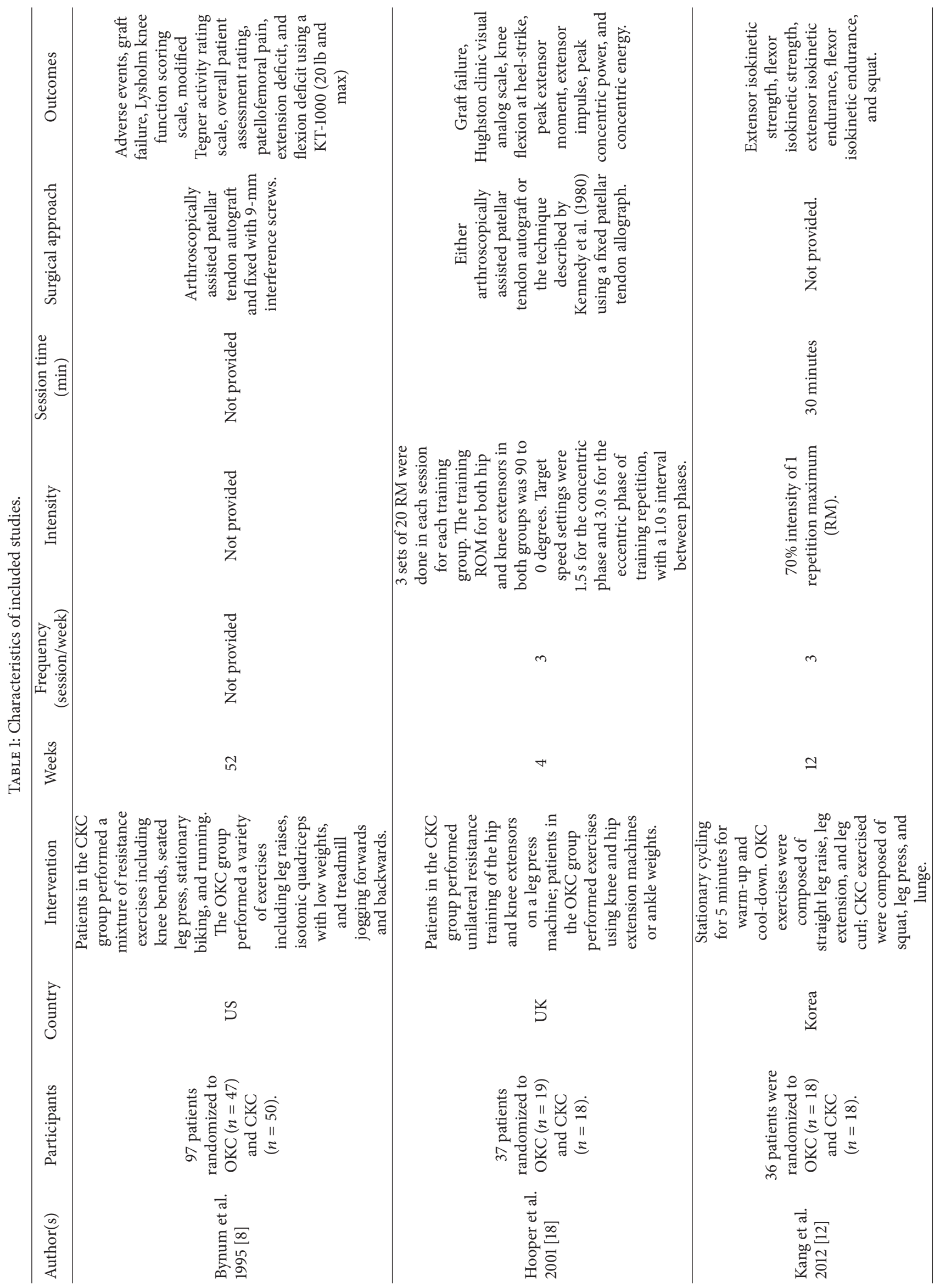




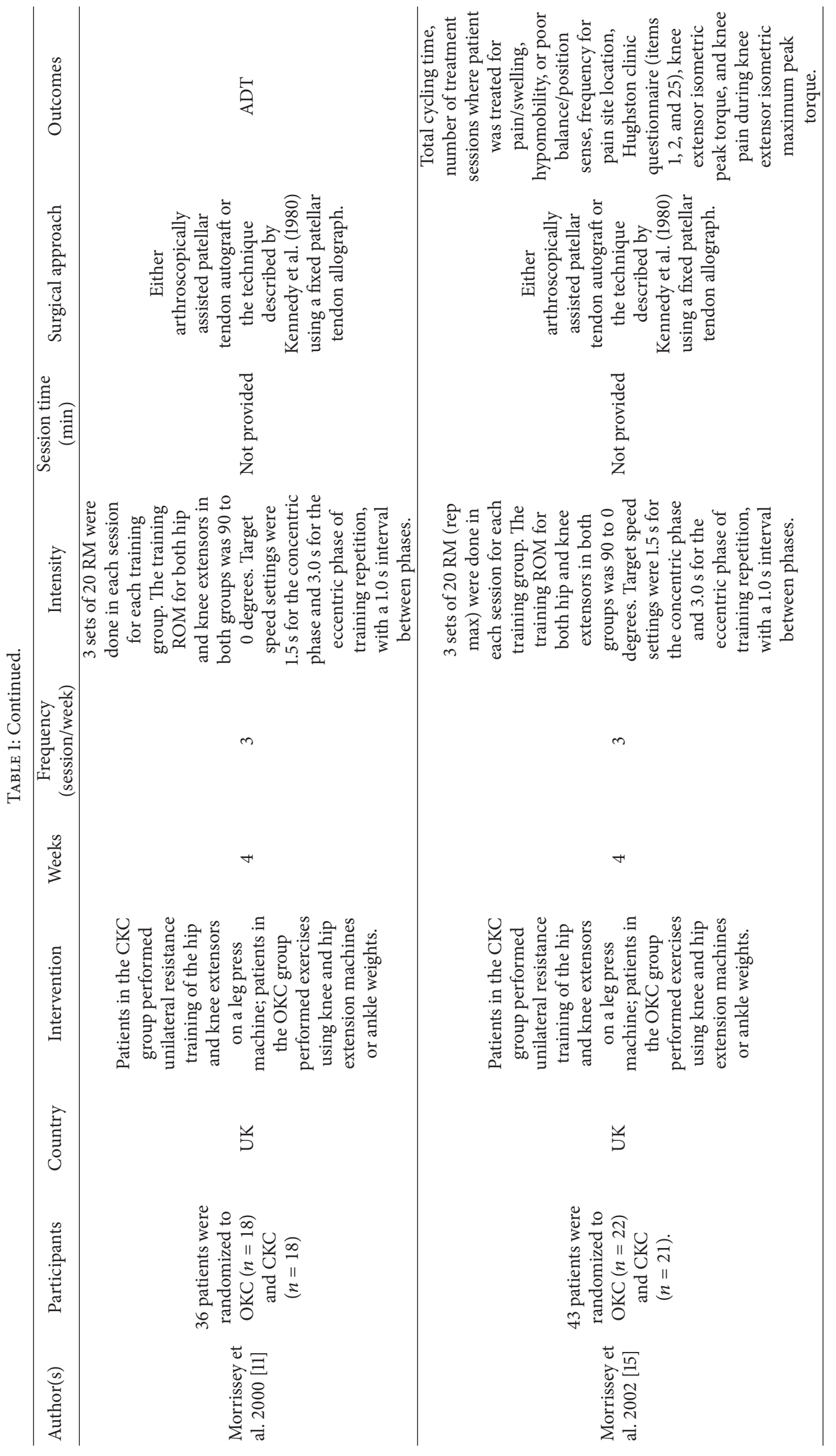




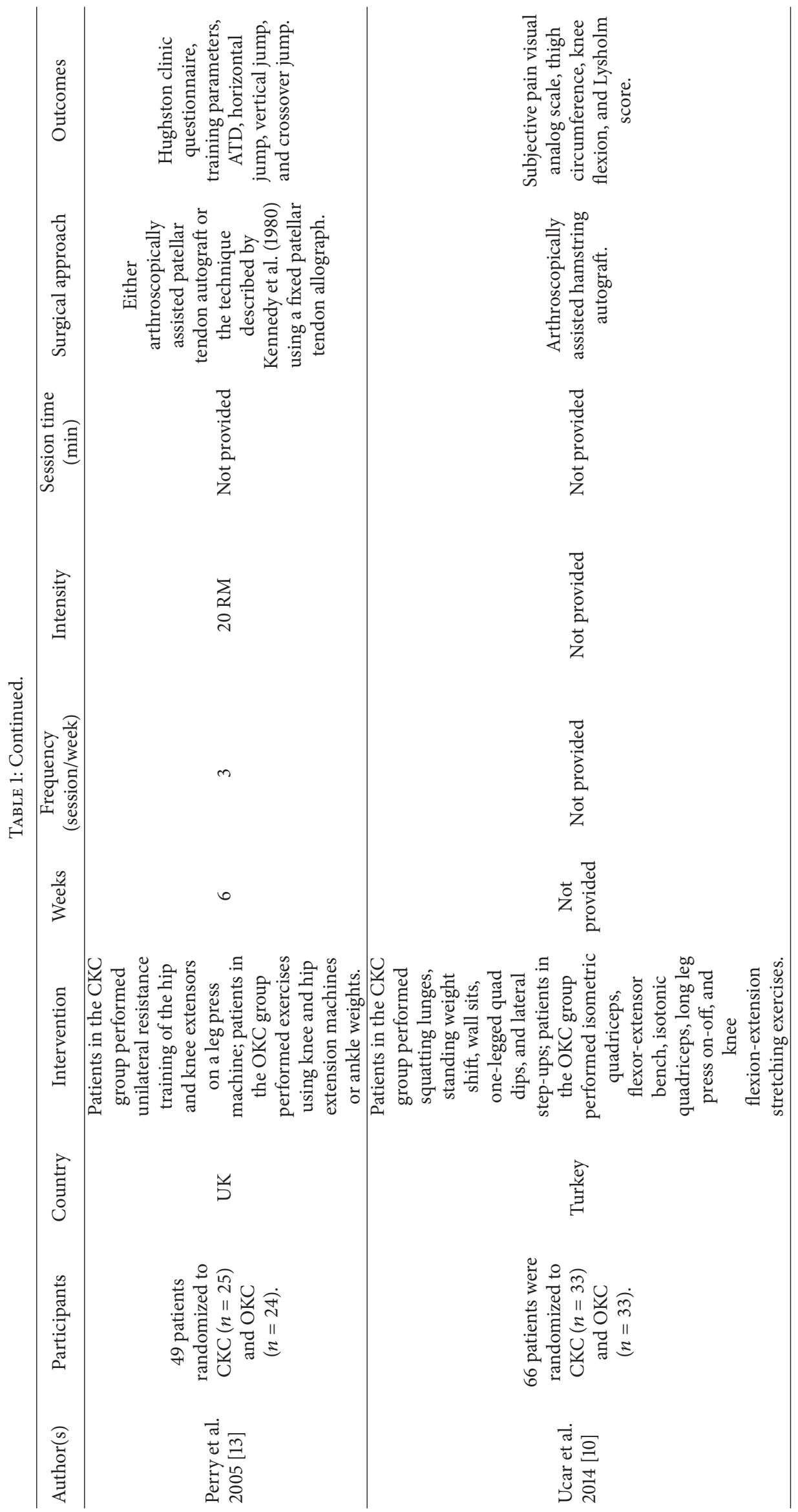


TABle 2: Excluded studies.

\begin{tabular}{lc}
\hline Study & Reason for exclusion \\
\hline Bird and Bulkeley 2010 [19] & Review paper \\
Chrzan et al. 2013 [20] & Intervention was not OKC versus CKC \\
Davis 1996 [21] & Population was not postsurgical \\
Dolan 2010 [22] & Not an RCT \\
Fitzgerald 1997 [23] & Not an RCT \\
Hooper et al. 2002 [24] & Intervention was not OKC versus CKC \\
Jenkins et al. 1997 [25] & Population was not postsurgical \\
Keays et al. 2013 [26] & Population was not postsurgical \\
Laboute et al. 2008 [27] & Intervention was not OKC versus CKC \\
Lage et al. 1995 [28] & Population was not postsurgical \\
Mikkelsen et al. 2000 [29] & Intervention was not OKC versus CKC \\
Neeter et al. 2006 [30] & Intervention was not OKC versus CKC \\
Perry et al. 2005 [13] & Population was not postsurgical \\
Petschnig and Baron 1997 [31] & Intervention was not OKC versus CKC \\
Rennison 1996 [32] & Not an RCT \\
Ross et al. 2001 [33] & Not an RCT \\
Tagesson et al. 2008 [34] & Population was not postsurgical \\
\hline
\end{tabular}

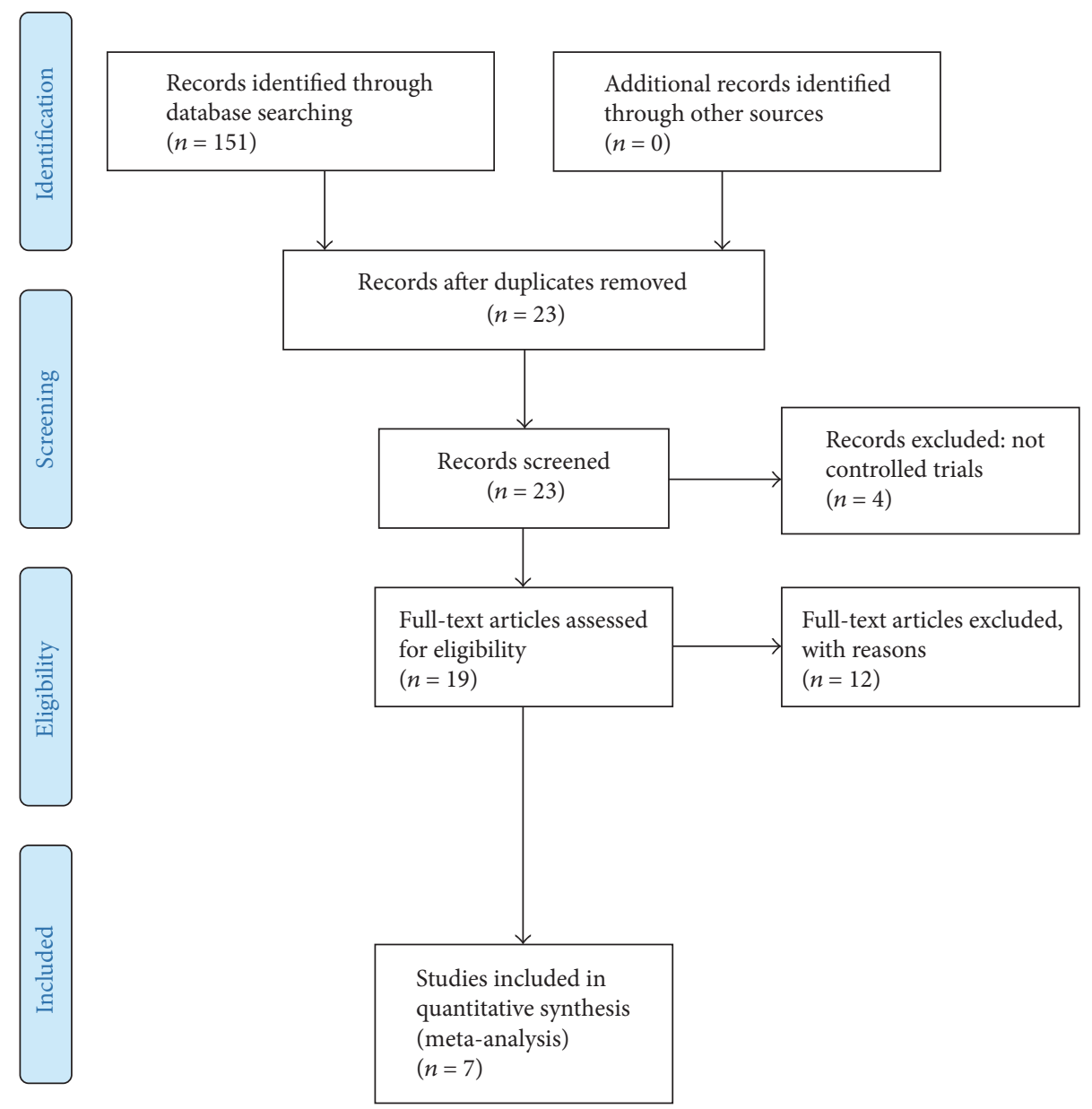

Figure 1: CONSORT statement. 


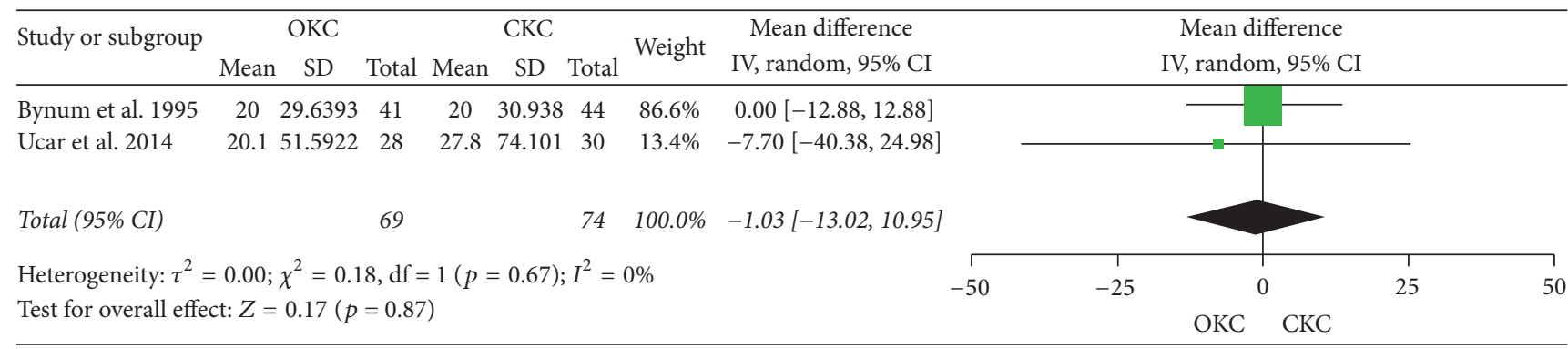

FIGURE 2: Lysholm score.

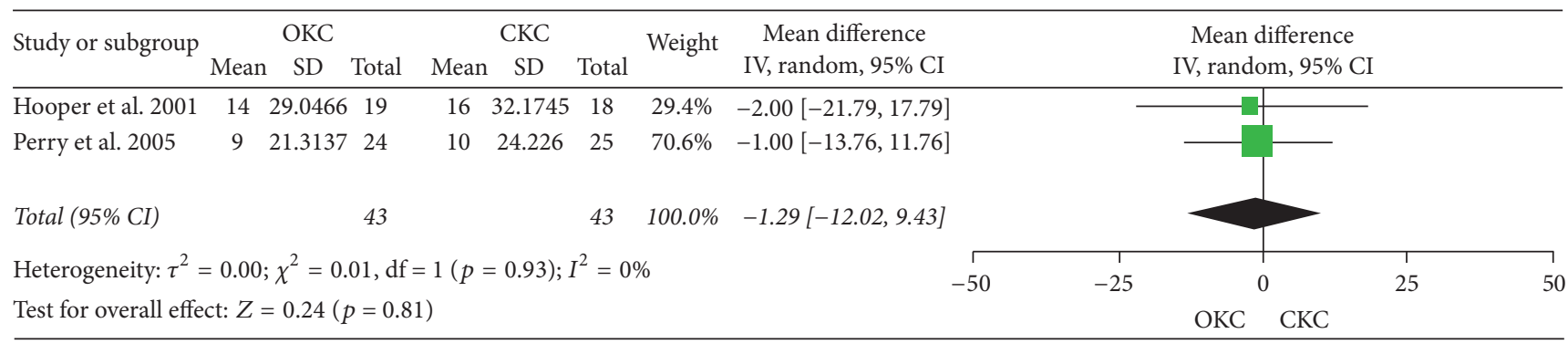

FIgURE 3: Hughston score.

3.2. Systematic Review. Insufficient data existed to conduct meta-analyses for other outcome measures, so these are systematically described here.

3.2.1. Patellofemoral Joint Pain. Although Bynum et al., 1995 [8], reported significantly less pain in CKC compared to $\mathrm{OKC}$, they also reported no significant difference of Tegner activity scale score [9] in OKC versus CKC groups. Aside from varying levels of activity between groups, the authors also stated that these findings are not likely to be clinically significant. Ucar et al., 2014, reported more pain with OKC, but level of statistical significance was unclear [10]. Morrissey et al., 2000, found no significant difference between the groups in regard to knee extensor knee pain [11].

3.2.2. Knee Extensor Strength. Kang et al., 2012, reported that isokinetic strength, isokinetic endurance, and squat strength improved significantly after both interventions $(p<0.05)$, but changes in isokinetic strength and isokinetic endurance of the extensor muscles were significantly greater in the $\mathrm{OKC}$ group than the CKC group $(p<0.05)$ [12]. In contrast Morrisey et al., 2000, found no difference between groups [11].

3.2.3. Knee Anterior Laxity. Morrissey et al., 2000 [11], and Perry et al., 2005 [13], both found no statistically significant difference between OKC and CKC groups on Anterior Tibial Displacement test.

3.2.4. Active Knee Flexion. Ucar et al., 2014, reported knee flexion was greater in the CKC group than the OKC group; however they do not report whether these differences were statistically significant [10].
3.3. Study Quality. The TESTEX scale [6] of study quality revealed a median score of 7 (out of a possible 14). As there was no sedentary control group in included studies TESTEX was scored out of 14 not usual 15. Study quality items that were not exhibited by more than $50 \%$ of studies were allocation concealment ( 1 study), assessor blinding ( 0 studies), intention of treatment analysis (0 studies), point measures and measures of variability reporting (3 studies), relative exercise intensity review (1 study), and exercise volume and expenditure (1 study).

\section{Discussion}

Our systematic review and meta-analysis suggest that a few direct, randomized, controlled comparisons of $\mathrm{OKC}$ and $\mathrm{CKC}$ knee extensor resistance training after anterior cruciate ligament reconstruction exist in the published literature. Both the statistical and descriptive analyses suggest both open and closed kinetic chain exercises are beneficial interventions for people with reconstructed ACLs. Moreover, of the few studies that do exist, our meta-analyses showed no significant difference between OKC and CKC for pain scores, knee extensor strength, laxity, and knee flexion.

4.1. Pain. Some previous work has asserted that CKC rehabilitative exercises are safer in terms of pain [14]. We also found evidence to the contrary [15]. Overall we found that there is insufficient evidence within the literature pool to either support or dispel this opinion. The meta-analyses of pain are unable to discern between $\mathrm{OKC}$ and $\mathrm{CKC}$ exercise rehabilitation programming. There are several explanations why no significant difference was seen. First, it may be that the questionnaire is not sensitive enough to detect small changes 
in pain and function. Second, the statistical power of the analyses is limited by a small number of studies with small sample size and the addition of future studies will determine if there is truly a difference. The final explanation is that there is actually no difference in pain experienced by people undertaking $\mathrm{OKC}$ versus CKC.

4.2. Strength. We were unable to pool data for an analysis of knee extensor strength. Descriptive analysis of the included studies of our systematic review was also inconclusive. A study of normal anterior cruciate ligament subjects was also unable to show either OKC or CKS to be superior for improving knee strength [16].

4.3. Knee Anterior Laxity. We were unable to pool data for analysis of knee extensor strength and descriptive analysis of included studies did not provide further clarity on superiority of either an OKC or CKC approach.

4.4. Knee Flexion. Only one study reported on the outcome measure knee flexion and this work claimed OKC exercise to be superior for restoring knee flexion after ACL reconstructive surgery. This work did not however provide statistical analyses to support the superiority of OKC. Further work on restoration of knee flexion after ACL surgery is clearly required.

The systematic review part of our work does appear to provide a stronger case for improvements in knee extensor strength with open kinetic chain exercises versus closed chain exercise. In contrast active knee flexion appears to be superior with closed kinetic chain exercises in comparison to open kinetic chain exercises. From our work, we cannot discern if patellofemoral joint pain and knee laxity are unaffected by the type of exercise rehabilitation used, shedding doubt as to whether OKC exercises are more likely to cause graft failure.

It is quite clear that the number of studies reporting meaningful data is currently limited. After conducting our search and analyses it is also apparent that the sample size of all studies is limited, with the exception of Bynum et al's 1995 study [8]. It is therefore surprising that such a strong contention exists that CKC exercises are optimal. Based upon the evidence presented in our work, one can but surmise that any preference for either OKC or CKC would be subjective at best. A large, well-designed, randomized, controlled trial is required to clarify this matter. There are significant advances in the diagnostic power of tissue imaging, such as Magnetic Resonance Imaging, since Bynum's 1995 work. The main purpose of MRI in patients with an ACL injury lies in accurate diagnosis or exclusion of a tear in patients with equivocal physical examination findings. ACL injury management is critically dependent on accurate diagnosis of other coexisting knee internal lesions, in particular tears of the lateral collateral ligament (LCL), posterior cruciate ligament (PCL), and the menisci [17]. It would also be prudent that future trials incorporate outcome measurements that are quantitative assessments, based upon measurements conducted by imaging experts who are blinded to participant allocations.
Limitations. The small volume of data precluded metaanalyses in several outcome measures and in those that were conducted statistical power was limited. Many of the outcome measures are self-reported subjective measurements. The existing literature has yet to optimize use of advances in imaging techniques. Study quality assessment suggests that future study designs could be more robust and strictly implement assessor blinding. Both of these enhancements would be likely to produce data more meaningful than those currently existing.

\section{Conclusions}

Based upon existing published data it is difficult to make a case for superiority of either open or closed chain kinetic exercise rehabilitation after ACL reconstruction. Metaanalyses failed to show a benefit of either type of exercise in terms of joint laxity and patellofemoral joint pain scores. While there was weak evidence that open chain exercises are better for improving knee extensor strength, this is countered by weak evidence for better active knee flexion in closed chain activities.

\section{Conflicts of Interest}

The authors declare that they have no conflicts of interest.

\section{References}

[1] A. V. Lukianov, J. Gillquist, W. A. Grana, and K. E. DeHaven, "An anterior cruciate ligament (ACL) evaluation format for assessment of artificial or autologous anterior cruciate reconstruction results," Clinical Orthopaedics and Related Research, vol. 218, pp. 167-180, 1987.

[2] R. Glass, J. Waddell, and B. Hoogenboom, "The effects of open versus closed kinetic chain exercises on patients with acl deficient or reconstructed knees: a systematic review," North American Journal of Sports Physical Therapy, vol. 5, no. 2, pp. 74-84, 2010.

[3] H. J. Yack, C. E. Collins, and T. J. Whieldon, "Comparison of closed and open kinetic chain exercise in the anterior cruciate ligament-deficient knee," American Journal of Sports Medicine, vol. 21, no. 1, pp. 49-54, 1993.

[4] B. D. Beynnon and B. C. Fleming, "Anterior cruciate ligament strain in-vivo: a review of previous work," Journal of Biomechanics, vol. 31, no. 6, pp. 519-525, 1998.

[5] F. Flandry, J. P. Hunt, G. C. Terry, and J. C. Hughston, "Analysis of subjective knee complaints using visual analog scales," American Journal of Sports Medicine, vol. 19, no. 2, pp. 112-118, 1991.

[6] N. A. Smart, M. Waldron, H. Ismail et al., "Validation of a new tool for the assessment of study quality and reporting in exercise training studies: TESTEX," International Journal of EvidenceBased Healthcare, vol. 13, no. 1, pp. 9-18, 2015.

[7] J. P. T. Higgins, J. J. Deeks, and D. G. Altman, "Measuring inconsistency in meta-analyses," British Medical Journal, vol. 327, no. 7414, pp. 557-560, 2003.

[8] E. B. Bynum, R. L. Barrack, and A. H. Alexander, "Open Versus Closed Chain Kinetic Exercises After Anterior Cruciate Ligament Reconstruction: A Prospective Randomized Study," 
The American Journal of Sports Medicine, vol. 23, no. 4, pp. 401406, 1995.

[9] K. K. Briggs, M. S. Kocher, W. G. Rodkey, and J. R. Steadman, "Reliability, validity, and responsiveness of the Lysholm knee score and tegner activity scale for patients with meniscal injury of the knee," Journal of Bone and Joint Surgery - Series A, vol. 88, no. 4, pp. 698-705, 2006.

[10] M. Ucar, I. Koca, M. Eroglu et al., "Evaluation of open and closed kinetic chain exercises in rehabilitation following anterior cruciate ligament reconstruction," Journal of Physical Therapy Science, vol. 26, no. 12, pp. 1875-1878, 2014.

[11] M. C. Morrissey, Z. L. Hudson, W. I. Drechsler, F. J. Coutts, P. R. Knight, and J. B. King, "Effects of open versus closed kinetic chain training on knee laxity in the early period after anterior cruciate ligament reconstruction," Knee Surgery, Sports Traumatology, Arthroscopy, vol. 8, no. 6, pp. 343-348, 2000.

[12] H. Kang, J. Jung, and J. Yu, "Comparison of strength and endurance between open and closed kinematic chain exercises after anterior cruciate ligament reconstruction: Randomized control trial," Journal of Physical Therapy Science, vol. 24, no. 10, pp. 1055-1057, 2012.

[13] M. C. Perry, M. C. Morrissey, J. B. King, D. Morrissey, and P. Earnshaw, "Effects of closed versus open kinetic chain knee extensor resistance training on knee laxity and leg function in patients during the 8- to 14-week post-operative period after anterior cruciate ligament reconstruction," Knee Surgery, Sports Traumatology, Arthroscopy, vol. 13, no. 5, pp. 357-369, 2005.

[14] E. Witvrouw, R. Lysens, J. Bellemans et al., "Which factors predict outcome in the treatment program of anterior knee pain?" Scandinavian Journal of Medicine and Science in Sports, vol. 12, no. 1, pp. 40-46, 2002.

[15] M. C. Morrissey, W. I. Drechsler, D. Morrissey, P. R. Knight, P. W. Armstrong, and T. B. McAuliffe, "Effects of distally fixated versus nondistally fixated leg extensor resistance training on knee pain in the early period after anterior cruciate ligament reconstruction," Physical Therapy, vol. 82, no. 1, pp. 35-43, 2002.

[16] L. R. Felicio, A. D. P. Baffa, R. F. Liporacci, M. C. Saad, A. S. D. Oliveira, and D. Bevilaqua-Grossi, "Analysis of patellar stabilizers muscles and patellar kinematics in anterior knee pain subjects," Journal of Electromyography and Kinesiology, vol. 21, no. 1, pp. 148-153, 2011.

[17] B. Munk, F. Madsen, E. Lundorf et al., "Clinical magnetic resonance imaging and arthroscopic findings in knees: A comparative prospective study of meniscus anterior cruciate ligament and cartilage lesions," Arthroscopy, vol. 14, no. 2, pp. 171-175, 1998.

[18] D. M. Hooper, M. C. Morrissey, W. Drechsler, D. Morrissey, and J. King, "Open and closed kinetic chain exercises in the early period after anterior cruciate ligament reconstruction: Improvements in level walking, stair ascent, and stair descent," American Journal of Sports Medicine, vol. 29, no. 2, pp. 167-174, 2001.

[19] S. Bird and P. Bulkeley, "Open chain knee extension exercise compared to closed chain squat exercise-which is more effective in the rehabilitation of acl injuries?" Journal of Australian Strength Conditioning, vol. 18, no. 1, pp. 12-15, 2010.

[20] D. Chrzan, D. Kusz, W. Bołtuć, A. Bryła, and B. Kusz, "Subjective assessment of rehabilitation protocol by patients after ACL reconstruction - Preliminary report," Ortopedia Traumatologia Rehabilitacja, vol. 15, no. 3, pp. 215-225, 2013.
[21] J. Davis, "Knee (ACL) trauma rehabilitation: open versus closed kinetic chain exercises in an anterior cruciate ligament deficient knee of a female high school basketball player," Journal of Bodywork Movement Therapies [serial on the Internet], vol. 1, no. 1, pp. 58-61, Oct 1996, Available from: CINAHL Complete.

[22] M. G. Dolan, "Open kinetic chain versus closed kinetic chain exercise after ACL injury," Athletic Therapy Today, vol. 15, no. 3, pp. 8-10, 2010.

[23] G. K. Fitzgerald, "Open versus closed kinetic chain exercise: Issues in rehabilitation after anterior cruciate ligament reconstructive surgery," Physical Therapy, vol. 77, no. 12, pp. 1747-1754, 1997.

[24] D. M. Hooper, H. Hill, W. I. Drechsler, and M. C. Morrissey, "Range of motion specificity resulting from closed and open kinetic chain resistance training after anterior cruciate ligament reconstruction," Journal of Strength and Conditioning Research, vol. 16, no. 3, pp. 409-415, 2002.

[25] W. L. Jenkins, S. W. Munns, G. Jayaraman, K. L. Wertzberger, and K. Neely, "A measurement of anterior tibial displacement in the closed and open kinetic chain," Journal of Orthopaedic and Sports Physical Therapy, vol. 25, no. 1, pp. 49-56, 1997.

[26] S. L. Keays, M. Sayers, D. B. Mellifont, and C. Richardson, “Tibial displacement and rotation during seated knee extension and wall squatting: a comparative study of tibiofemoral kinematics between chronic unilateral anterior cruciate ligament deficient and healthy knees," Knee, vol. 20, no. 5, pp. 346-353, 2013.

[27] E. Laboute, L. Savalli, T. Lefesvre, P. Puig, and P. Trouve, "Interest of an iterative specialized rehabilitation after an anterior cruciate ligament reconstruction in high level sport athletes," Revue de Chirurgie Orthopedique et Reparatrice de l'Appareil Moteur, vol. 94, no. 6, pp. 533-540, 2008.

[28] K. Lage, S. White, and H. Yack, "The effects of unilateral knee immobilization on lower extremity gait mechanics. / Effets d' une immobilisation unilaterale du genou sur les mecanismes de la demarche pour les membres inferieurs," Medicine Science In Sports Exercise, vol. 27, no. 1, pp. 8-14, 1995.

[29] C. Mikkelsen, S. Werner, and E. Eriksson, "Closed kinetic chain alone compared to combined open and closed kinetic chain exercises for quadriceps strengthening after anterior cruciate ligament reconstruction with respect to return to sports: A prospective matched follow-up study," Knee Surgery, Sports Traumatology, Arthroscopy, vol. 8, no. 6, pp. 337-342, 2000.

[30] C. Neeter, A. Gustavsson, P. Thomeé, J. Augustsson, R. Thomeé, and J. Karlsson, "Development of a strength test battery for evaluating leg muscle power after anterior cruciate ligament injury and reconstruction," Knee Surgery, Sports Traumatology, Arthroscopy, vol. 14, no. 6, pp. 571-580, 2006.

[31] R. Petschnig and R. Baron, "Assessment of metabolic response and functional changes after anterior cruciate ligament surgery," Medicine and Science in Sports and Exercise, vol. 29, no. 1, pp. 39, 1997.

[32] M. Rennison, "Open versus closed chain kinetic exercises after anterior cruciate ligament reconstruction. A prospective randomized study.," The American journal of sports medicine, vol. 24, no. 1, p. 125, 1996.

[33] M. D. Ross, C. R. Denegar, and J. A. Winzenried, "Implementation of Open and Closed Kinetic Chain Quadriceps Strengthening Exercises after Anterior Cruciate Ligament Reconstruction," Journal of Strength and Conditioning Research, vol. 15, no. 4, pp. 466-473, 2001. 
[34] S. Tagesson, B. Öberg, L. Good, and J. Kvist, "A comprehensive rehabilitation program with quadriceps strengthening in closed versus open kinetic chain exercise in patients with anterior cruciate ligament deficiency: A randomized clinical trial evaluating dynamic tibial translation and muscle function," American Journal of Sports Medicine, vol. 36, no. 2, pp. 298-307, 2008. 


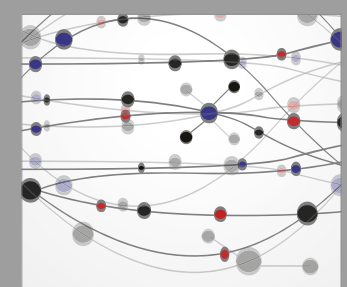

The Scientific World Journal
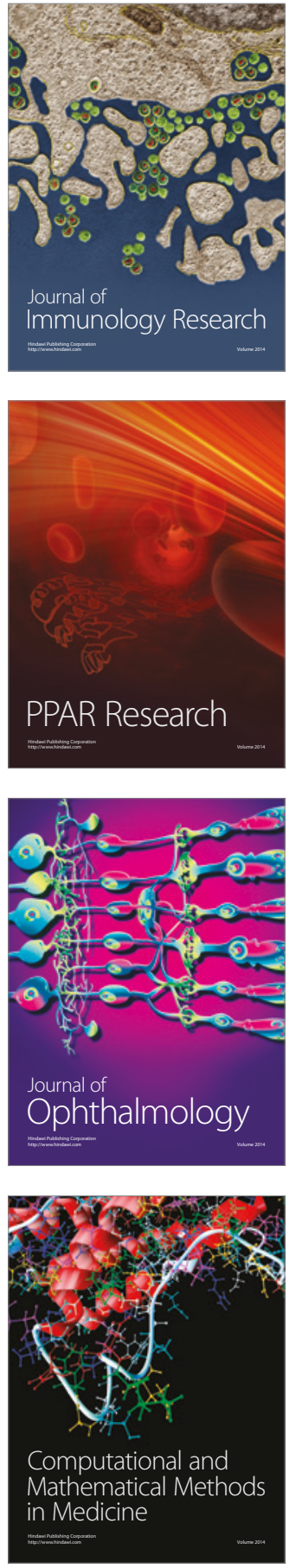

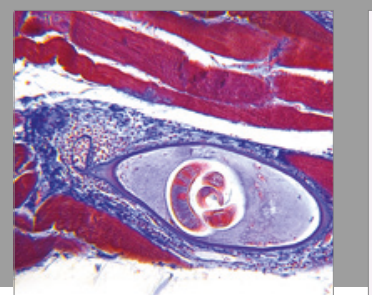

Gastroenterology Research and Practice
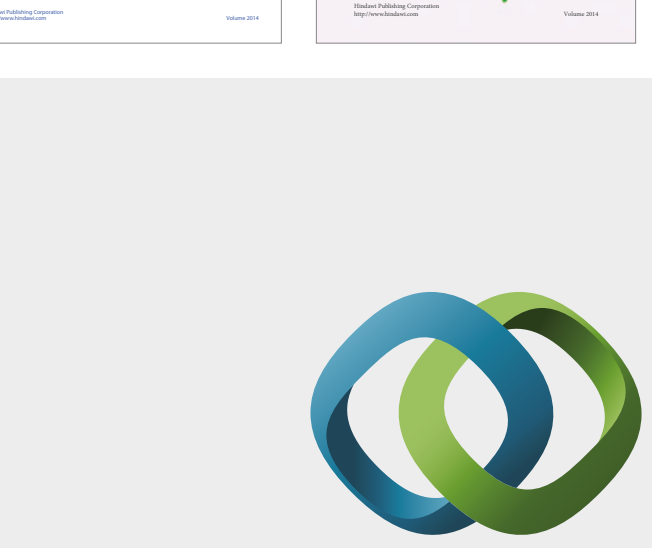

\section{Hindawi}

Submit your manuscripts at

https://www.hindawi.com
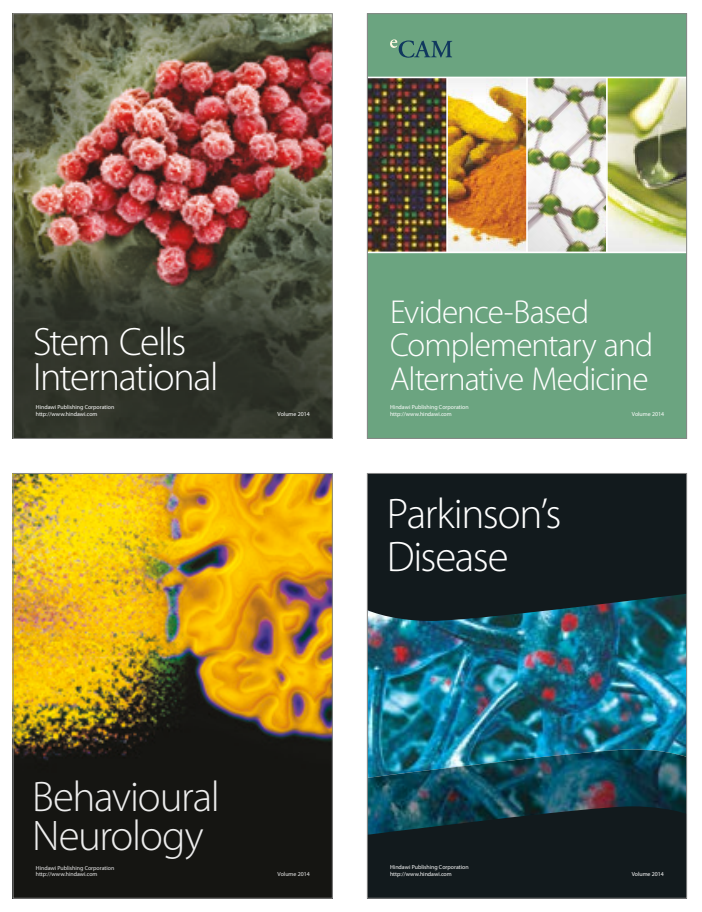
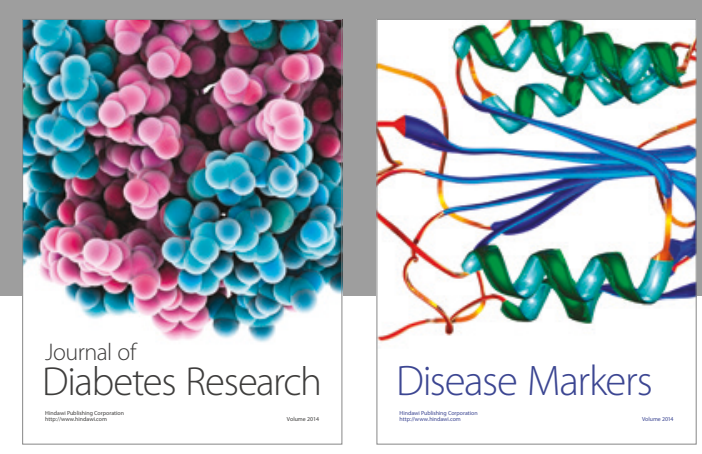

Disease Markers
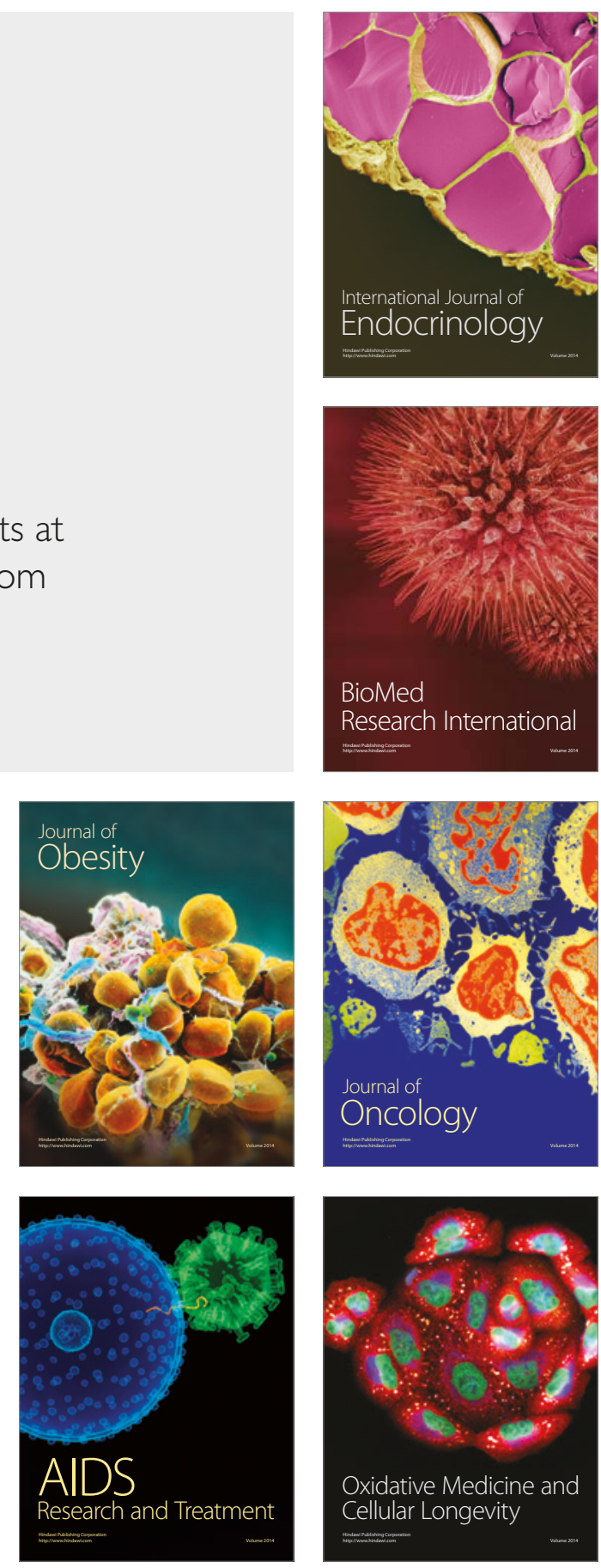